\title{
Pandemic H1N1 influenza A infection and (atypical) HUS - more than just another trigger?
}

\author{
Upton Allen • Christoph Licht
}

Received: 22 August 2010 /Accepted: 7 September 2010 /Published online: 7 November 2010

(C) IPNA 2010

\begin{abstract}
Atypical hemolytic uremic syndrome (aHUS) is caused by mutations resulting in an exceedingly active alternative complement pathway. While today more than half a dozen genes are involved in aHUS pathology, only about $50 \%$ of carriers precipitate the disease. The reason for this phenomenon remains unclear, and triggering events like intercurrent infections have been postulated. In this context, reports on the development of (a)HUS in patients concomitantly diagnosed with pandemic H1N1 influenza A (pH1N1) infection are of great interest. They establish-for the first time in the literature - the link between aHUS and pH1N1 infection. While illnesses associated with pH1N1 infections during the recent pandemics were generally mild, secondary bacterial infections (e.g. Streptococcus pneumoniae) are known in patients with influenza A infections to not only aggravate the disease course, but also serve as a possible HUS trigger. Assuming pH1N1 was the cause of HUS in the cases reported here, it remains an interesting but unanswered hypothesis whether an underlying complement defect served as a susceptibility factor, at least in a subgroup of patients. In the future, pH1N1, but also pH1N1-associated, bacterial infections
\end{abstract}

\footnotetext{
U. Allen

Division of Infectious Diseases, The Hospital for Sick Children,

555 University Avenue,

Toronto, ON M5G 1X8, Canada

U. Allen · C. Licht

University of Toronto,

Toronto, ON, Canada

C. Licht $(\bowtie)$

Division of Nephrology, The Hospital for Sick Children,

555 University Avenue,

Toronto, ON M5G 1X8, Canada

e-mail: christoph.licht@sickkids.ca
}

will have to be considered in (a)HUS patients, and further studies will be required to examine the role of the complement system in this condition.

Keywords H1N1 · Hemolytic uremic syndrome · Pneumococcal infection

\section{Background}

Hemolytic uremic syndrome (HUS) is a thrombotic microangiopathy (TMA) that is defined by hemolytic anemia, thrombocytopenia, and renal failure [1]. In its typical form, HUS is caused by exotoxins (e.g. Shiga or Shiga-like toxin) of certain bacteria (e.g. Escherichia coli O157:H7 or Shigella dysenteriae type 1), which enter the body via the gastrointestinal tract and gain access to the circulation through the inflamed bowel walls. Subsequently, these toxins bind to specific receptors on the glomerular endothelium, resulting in endothelial cell death and subsequent activation of the clotting cascade including the formation of thromboemboli and occlusion of the glomerular microcapillaries $[2,3]$.

By contrast, atypical HUS (aHUS) is caused by defects in the regulation of the alternative complement pathway on vascular endothelial cells. In brief, the alternative complement pathway - different from the inducible classical or mannoselectin pathways - is constantly active on surfaces, thus requiring a potent negative regulation system to ensure siteand time-directed activation and to prevent disease causing over-activity. Complement control is provided by a multilayered system of soluble (e.g. complement factor $\mathrm{H}, \mathrm{CFH}$; and complement factor I, CFI) and membrane-anchored (e.g. membrane cofactor protein, $\mathrm{MCP} / \mathrm{CD} 46$; thrombomodulin, THBD/CD141) proteins. While mutations in these regulators 
result in loss of control of the alternative $\mathrm{C} 3$ convertase, $\mathrm{C} 3 \mathrm{bBb}$, mutations in complement factor $\mathrm{B}$ (CFB) or $\mathrm{C} 3$ result in gain of the function of this enzyme. Today, $50-60 \%$ of aHUS cases can be explained by one (or a combination) of these mutations or by the presence of inhibiting autoantibodies (i.e. anti-CFH autoantibodies) [4].

While according to these categories typical HUS can be caused by infections with Escherichia coli or Shigella dysenteriae (but also with other bacteria like Streptococcus pneumoniae or viruses like influenza A) [5], infectious triggers for the atypical form of HUS have been postulated in the literature, but not yet been defined. However, as only about $50 \%$ of individuals carrying disease causing mutations precipitate aHUS, concomitant infections as triggers for disease onset are an upcoming theme in the current literature.

\section{Pandemic influenza A 2009 (H1N1) infection and HUS}

In the above context, a series of four letters published in the current issue of Pediatric Nephrology is of great interest [6-9]. These letters report on the development of HUS in patients who were concomitantly diagnosed with pandemic H1N1 influenza A (pH1N1) infection. All four reports establish a link between aHUS and pH1N1 infection, an observation that has not been previously reported in the literature. This finding is of clinical relevance, as HUS has to now be considered a possible complication of $\mathrm{pH} 1 \mathrm{~N} 1$ infection. Consequently, pH1N1 (and influenza $\mathrm{A}$ in general) should be added to the infectious diseases of HUS patients.

In one of the presented cases [6] the authors investigated key regulators of the alternative complement pathway (i.e. $\mathrm{CFH}$ and $\mathrm{CFI}$ ), thus indicating that they considered an underlying complement defect a possible susceptibility factor for the H1N1 infection. While in this case no quantitative abnormality in the examined complement regulators could be detected, the role of complement defects in H1N1-related HUS can obviously not yet be defined, and - given the diagnostic and therapeutic implications - it will be more than just an academic exercise to aim to clarify whether H1N1-related HUS has to be considered in the context of complement defects or not via future studies.

There are three types of influenza viruses (A, B, and C) of which $\mathrm{A}$ and $\mathrm{B}$ are more known to cause illnesses in humans. Infants and young children are among the groups that are known to be at highest risk of severe outcomes from influenza illness. The pandemic strain of influenza A 2009 (H1N1) became a global concern because it emerged as a new virus as a result of the reassortment of influenza A viruses, to which humans had no significant immunity $[10,11]$. In this regard, influenza A viruses have eight separate gene segments. This allows the viruses to mix and reassort themselves to create new viral strains. The pandemic strain of H1N1 (pH1N1) was a quadruple reassortment virus with origins from swine (two strains), human and avian influenza A viruses [11-13]. Non-pandemic strains of H1N1 exist and are known to cause seasonal influenza A illness.

The first cases of the novel pandemic influenza A (H1N1) 2009 were recognized in Spring 2009 [12, 13]. Two waves of illness were observed in some regions [14]. In August 2010, the pandemic was officially declared over by the World Health Organization (WHO). Overall, the illnesses associated with $\mathrm{pH} 1 \mathrm{~N} 1$ were generally mild. There have been no recognized genetic markers associated with virulence characteristics that are distinct from seasonal influenza A. It is known that in a minority of cases, more severe illnesses with complications were reported. To this end, one of the known complications of pH1N1 influenza A infection is secondary bacterial infections. Invasive infections due to Streptococcus pneumoniae, Staphylococcus aureus, group A Streptococcus and other bacterial pathogens may occur $[15,16]$. Notably, the relationship between influenza A and Streptococcus pneumoniae is well researched, with evidence of a bi-directional interaction between these two pathogens [17]. If co-infection is present, this makes it challenging in some patients to determine which of these pathogens is responsible for the severe pulmonary and/or septic-related complications in the setting of influenza illness.

\section{pH1N1—a trigger for HUS?}

In considering the possible relationship between $\mathrm{pH} 1 \mathrm{~N} 1$ and HUS, it is necessary to consider the following questions. First, is there biological plausibility? Second, is there clear evidence of cause and effect? Third, is aHUS triggered by $\mathrm{pH} 1 \mathrm{~N} 1$ or a complication of pH1N1? Biological plausibility exists as outlined above, although the precise mechanism by which pH1N1 (or for that matter influenza A) causes HUS has not been definitively established. A cause and effect relationship can be inferred if there is good evidence that indicates an absence of other known triggers of HUS. The presence of multiple independent reports enhances the credibility that pH1N1 influenza A is associated with HUS. However, one should be reminded that $\mathrm{pH} 1 \mathrm{~N} 1$ influenza A could lead to the development of pneumococcal pneumonia and sepsis, which in turn could also lead to HUS. In this context, the relationship between HUS and pneumococcal infection is well established [3]. The report by Trachtman et al. [9] suggests that pneumococcal pneumonia was unlikely, although not definitively ruled out as a cause. In the Trachtman et al. report [9] as well as those by Printza et al. [7] and Golubovic et al. [8], 
antibiotics were part of the patients' treatment. These antibiotics (ceftriaxone with or without vancomycin) would be effective in treating pneumococcal infections. However, in the report by Caltik et al. [6], there is no mention of antibiotics. This would lessen the probability that there was pneumococcal co-infection and increase the probability that pH1N1 was indeed the trigger.

Assuming pH1N1 was the cause of aHUS in the patients reported in the above four reports, the evidence is lacking at this point to indicate if there is more of a propensity toward HUS with pH1N1 compared with seasonal H1N1 or other strains of influenza A viruses.

\section{Summary}

This series of four case reports links for the first time in the literature $\mathrm{pH} 1 \mathrm{~N} 1$ influenza $\mathrm{A}$ infection or the combination of a pH1N1 influenza A infection and a subsequent Streptococcus pneumoniae infection and HUS. The suggestion of a complement defect as a susceptibility factor for pH1N1-triggered HUS seems compelling, but the specific role of the complement system remains to be established by future investigations.

\section{References}

1. Gasser C, Gautier E, Steck A, Siebenmann RE, Oechslin R (1955) Hemolytic-uremic syndrome: bilateral necrosis of the renal cortex in acute acquired hemolytic anemia. Schweiz Med Wochenschr 85:905-909

2. Noris M, Remuzzi G (2005) Hemolytic uremic syndrome. J Am Soc Nephrol 16:1035-1050

3. Besbas N, Karpman D, Landau D, Loirat C, Proesmans W, Remuzzi G, Rizzoni G, Taylor CM, Van de Kar N, Zimmerhackl LB (2006) A classification of hemolytic uremic syndrome and thrombotic thrombocytopenic purpura and related disorders. Kidney Int 70:423-431
4. Noris M, Remuzzi G (2009) Atypical hemolytic-uremic syndrome. N Engl J Med 361:1676-1687

5. Constantinescu AR, Bitzan M, Weiss LS, Christen E, Kaplan BS, Cnaan A, Trachtman H (2004) Non-enteropathic hemolytic uremic syndrome: causes and short-term course. Am J Kidney Dis 43:976-982

6. Caltik A, Akyuz SG, Erdogan O, Demircin G (2010) Hemolytic uremic syndrome triggered with a new pandemic virus: influenza A (H1N1). Pediatr Nephrol. doi:10.1007/s00467-010-1649-0

7. Printza N, Roilides E, Kotsiou M, Zafeiriou D, Hatzidimitriou V, Papachristou F (2010) Pandemic influenza A (H1N1) 2009associated hemolytic uremic syndrome. Pediatr Nephrol. doi:10.1007/s00467-010-1603-1

8. Golubovic E, Miljkovic P, Zivic S, Jovancic D, Kostic G (2010) Hemolytic uremic syndrome associated with novel influenza A H1N1 infection. Pediatr Nephrol. doi:10.1007/s00467-010-1687-7

9. Trachtman H, Sethna C, Epstein R, D'Souza M, Rubin L, Ginocchio C (2010) Atypical hemolytic uremic syndrome associated with H1N1 influenza A virus infection. Pediatr Nephrol. doi:10.1007/s00467-010-1636-5

10. Khiabanian H, Trifonov V, Rabadan R (2009) Reassortment patterns in swine influenza viruses. PLoS One 4:e7366

11. Scalera NM, Mossad SB (2009) The first pandemic of the 21 st century: a review of the 2009 pandemic variant influenza A (H1N1) virus. Postgrad Med 121:43-47

12. Centers for Disease Control and Prevention (CDC) (2009) Outbreak of swine-origin influenza A (H1N1) virus infectionMexico, March-April 2009. MMWR Morb Mortal Wkly Rep 58:467-470

13. Centers for Disease Control and Prevention (CDC) (2009) Update: swine influenza A (H1N1) infections - California and Texas, April 2009. MMWR Morb Mortal Wkly Rep 58:435-437

14. Satterwhite L, Mehta A, Martin GS (2010) Novel findings from the second wave of adult PH1N1 in the United States. Crit Care Med 38:2059-2061

15. Pediatrics AAo (2009) Influenza. In: Pickering LK, Baker CJ, Kimberlin DW, Long SS (eds) Red book: report on infectious diseases. American Academy of Pediatrics, Elk Grove Village, IL, pp 400-412

16. Thorner AR, Hirsch MS, McGovern BH (2010) Epidemiology, clinical manifestations, and diagnosis of pandemic H1N1 influenza ('swine influenza'). http://www.uptodate.com/home/content/ topic.do?topicKey=pulm_inf $/ 18836$

17. McCullers JA (2006) Insights into the interaction between influenza virus and pneumococcus. Clin Microbiol Rev 19:571582 